En Psychiatry and Neuroscience Update. From Translational Research to a Humanistic Approach - Volume III. Ed. PA Gargiulo, HL Mesones Arroyo. ISBN 9783319953595 Springer Nature Swiitzerland. 2019, pp 113-126

\title{
Heart rate variability: a tool to explore autonomic nervous system activity in health and disease
}

Daniel E Vigo (1,2); Leonardo Nicola Siri (3); Daniel P Cardinali $(1,4)$

(1) Chronophysiology Lab, Institute for Biomedical Research (BIOMED), Pontifical Catholic University of Argentina (UCA) and National Research Council (CONICET), Argentina.

(2) Research Group on Health Psychology, Faculty of Psychology and Educational Sciences, Katholieke Universiteit Leuven, Belgium.

(3) Southwest Regional Institute, Technological University, Fray Bentos, Uruguay

(4) Teaching and Research Department, Faculty of Medical Sciences, Pontifical Catholic University of Argentina (UCA), Argentina.

\begin{abstract}
It is accepted that several biological (genetic, physiological), psychological (personality, mood), social (family, work) and ecological (living environment) factors, interact to allow the preservation of quality of life and health. The autonomic nervous system (ANS) is structural and rhythmically interfaced between forebrain, internal and external environments, to regulate energy, matter and information exchanges, thus expressing the bio-psycho-social nature of the individual. During last year's strong evidence has been produced that demonstrate the influence of the ANS into beat to beat fluctuations of cardiac heart period. Stephen Hales was the first in describing the association between respiratory cycle and heart rate. However, it was not until the second half of the XX century that subtle relations between ANS activity and heart rate variability (HRV) were systematically explored in several physiological conditions, including physical activity, sleep and stress. In addition, autonomic imbalance may configure a final common pathway to increased morbidity and mortality from a host of physical, such as metabolic disorders and cardiovascular disease, and psychological conditions,
\end{abstract}


like anxiety or depression. This chapter will focus on the physiological mechanisms underlying $\mathrm{HRV}$, the methods for assessing it and the information that has provided about ANS activity in certain physiological and pathological situations.

Key words: Autonomic nervous system, Heart rate variability, Sleep, Stress, Complex diseases, Psychological disorders

\section{Historical perspective}

Within the different rhythmic processes observed in physiology, the heart rate has been one of the first to be studied. The earliest references come from Egyptian papyri (1550 $\mathrm{BC})$, where a dissertation on the heart and the veins in terms of measuring is found, probably with the meaning of "counting" the pulse, recognizing it as related to the heart. The first one who spoke of this calculation was Herophilus of Alexandria (3rd century BC) who used a clepsydra for this purpose. Subsequently, we find detailed descriptions of the pulse in the treatises of Galen (129 - c.199) on the subject. In the Renaissance, Galileo used his pulse to evaluate the oscillations of the pendulum, although it was Santorio (1561-1636) who used the pendulum to measure the frequency and regularity of the pulse. John Floyer (1649-1734) published an essay in 1707 (The Physician's pulse watch, or an essay to explain the old art of feeling the pulse and to improve it by the help of a pulse watch), where he described the invention of a portable clock with a second piece that could stop the watch. Thus, he could register pulse and respiration under several conditions. In 1733, Stephen Hales (1677-1761) described for the first time the relationship between the respiratory cycle and heart rate (Statical Essays, Vol. II Haemastatics), while in 1847, Carl Ludwig (1816-1895) was the first who documented the respiratory sinus arrhythmia. The recording of the electrical activity of the heart using galvanometers due to Willem Einthoven (1860-1927) in the late XIX century, and the further development by Jeff Holter (1914-1983) in early 1960s of portable devices capable of recording ambulatory ECG during long periods of time (24 h) allowed further research in the relation between heart rate variations with health and disease (1) . 


\section{Sources of HRV}

Heart rate is controlled by the periodic depolarization and repolarization of the cardiac pacemaker at the sinoatrial node, located on the posterior wall of the right atrium. Its steady intrinsic frequency range is 100 to 120 beats per minute. There are several neurohumoral factors that can modify this frequency, determining a lower or higher mean heart rate and beat to beat modifications of heartbeat duration. This latter phenomenon is known as "Heart Rate Variability" (HRV) (2). An intrinsic neural network within the cardiac fat pad provides local control of heart rate. This local network consists of sympathetic and parasympathetic neurons and interneuronal circuits. The spontaneous activity of these neurons even after cardiac denervation suggests an active role in the regulation of heart rate (3).

The origin of respiratory sinus arrhythmia (inspiratory tachycardia and expiratory bradycardia) is found in vagal modulation induced by the respiratory cycle on the heart. This mechanism determines that the duration of the heartbeat is modified following the respiratory rate, with cycles of 2.5 to 6.5 seconds in a healthy adult at rest, constituting HRV High Frequency component (HF: $0.15-0.4 \mathrm{~Hz}$ ) (Figure 1). Two mechanisms could explain this phenomenon. On the one hand, there would be a central coupling of the respiratory oscillator with autonomic centers of the brain stem in the ambiguous nucleus and other related areas. On the other hand, this mechanism would be mediated via a cardiopulmonary reflex. A decrease in intrapleural pressure during inspiration reduces intravascular pressure within the thorax and increases venous return, leading to increased volume in the atrium and right ventricle. Atrial mechanoreceptors would be activated by conducting information through sympathetic and parasympathetic pathways that would result in an increase in heart rate. Only the parasympathetic component of the efferent pathway would be involved because the sympathetic effect on the heart is too slow to follow the respiratory rate. This reflex was first suggested by Francis Bainbridge (1874-1921) in 1915 after observing the

increased heart rate in anesthetized dogs during volume infusions in the right atrium (4).

Spontaneous oscillations of blood pressure were first described by Siegmund Mayer (1842-1910) in 1875. The autonomic activity responsible for the genesis of these waves determines that the duration of heart beats is modified with cycles of 2.5 to $25 \mathrm{~s}$, constituting HRV Low Frequency component (LF: $0.04-0.15 \mathrm{~Hz}$ ) (Figure 1). The main mechanism responsible for these oscillations is the baroreflex. An increase in blood volume distends the baroreceptors of the aortic arch and the carotid sinus, which 
through the X-pair and the IX-pair respectively send information reaching the nucleus of the solitary tract. This stimulates the ambiguous nucleus producing an inhibition of the sympathetic preganglionic chain and stimulation of the dorsal motor nucleus of the vagus. The combined effects result in a decrease in heart rate. In addition, it is believed that there would be a direct central participation by which sympathetic autonomic oscillators would determine hemodynamic oscillations in the absence of peripheral stimuli (5).

Thermoregulation and several hormonal processes are possibly involved in slower variations of heart rate, with cycles of $25 \mathrm{~s}$ to 5 minutes, constituting HRV Very Low Frequency component (VLF: 0.0033 to $0.04 \mathrm{~Hz}$ ) (Figure 1). Central processing of the central and peripheral information about temperature is done in the anterior hypothalamus. In this region, neurons whose activity is affected by the thermal stimulation of the preoptic area or the spinal cord have been identified. Thermoregulation exerts an indirect effect on the heart rate through the sympathetic activation triggered by the cold. On the other hand, through a direct effect on the sinus node, cooling of the heart produces bradycardia, a mechanism used in cardiac surgery (6). With regard to hormonal factors, the renin - angiotensin - aldosterone system is thought to influence VLF oscillations. Angiotensin and other factors such as aldosterone would directly or indirectly produce fluctuations in vasomotor tone. These fluctuations in turn would determine, mainly through parasympathetic outflow, fluctuations in the heartbeat (7).

[Figure 1 here]

Heart Rate also exhibits a circadian rhythm, with ultra low frequency (ULF: $<0.0033$ $\mathrm{Hz}$ ) oscillations. This rhythm is partially originated in the basal forebrain and is dependent on the sleep-wake state. This region exerts control of cardiovascular autonomic function through widespread projections to the paralimbic cortex, amygdala, hypothalamus, and brainstem autonomic nuclei (8). In addition, the suprachiasmatic nuclei of the hypothalamus, considered the central pacemaker for circadian rhythms, regulate physiological functioning with cycles of about 24 hours that are adjusted to 24 $\mathrm{h}$ mainly by the information of ambient light. These nuclei have projections to the paraventricular hypothalamic nucleus, which in turn modulate ANS activity by sending input to major sites of ANS regulation. HF, LF, VLF and ULF frequency regions in the 
power spectra of cardiotachograms (see below), can be described as a "harmonic" (sin wave frequencies) regulation of HRV.

Furthermore, HRV shows also "unharmonic" components within its power spectrum, which indicate a nonlinear dynamic behavior derived from the complex interaction of external influences, internal input, autonomic tone, and central organization. Indeed, nonlinear features are characteristic of complex systems and the autonomic regulation of the heart can be described as one. As a complex system, it can be characterized by strange attractor's properties, fractal scaling, and the degree of entropy. Heart beats may be seen as the projection on a line of one trajectory of a dynamical system that will converge to a limited region in space or attractor. Strange attractor's properties include a similar structure at different scales (fractality) and sensitive dependence on initial conditions for the trajectories on them (chaotic behavior). Fractals are geometric objects that have similar structure at different scales. In analogous form, nonlinear processes show a statistically similar dynamic pattern at different scales. In other words, the irregular fluctuations seen at multiple time scales resemble each other, and the pattern of variation across multiple scales of measurement characterizes complex systems. Entropy is a measure of disorder or randomness of a system. Systems tend to evolve from statistically ordered unlikely configurations to statistically disordered more probable configurations (9-12).

\section{Methods for assessing HRV}

The simplest way to assess HRV is provided by the time domain methods (figure 1). In these methods the intervals between successive normal QRS complexes are measured ( $R R$ interval). Among others, simple time domain variables that can be calculated include the mean RR interval (RRM, ms); the standard deviation of all RR intervals (SDNN, ms), which gives a coarse quantification of HRV; and the square root of the mean of the squared differences between adjacent RR intervals (RMSSD, ms), which quantifies high frequency variations of HRV (Table 1) (13).

[Figure 2 here] 
Frequency domain methods or spectral analysis convert time domain information into frequency domain information (figure 3). Usually, the Discrete Fourier Transform is used for processing the signal, and the squared amplitude calculated for each frequency (the power spectral density) is obtained (13). By integration within suited frequency limits, the absolute spectral power $\left(\mathrm{ms}^{2}\right)$ can be calculated for each band: $\mathrm{HF}\left(\mathrm{ms}^{2}, 0.15 \mathrm{~Hz}-0.4 \mathrm{~Hz}\right)$; LF $\left(\mathrm{ms}^{2}, 0.04 \mathrm{~Hz}-0.15 \mathrm{~Hz}\right)$; VLF $\left(\mathrm{ms}^{2},<0.04 \mathrm{~Hz}\right.$ in short term recordings or $0.0033 \mathrm{~Hz}-0.04 \mathrm{~Hz}$ in long term recordings); ULF (ms ${ }^{2},<0.0033$ $\mathrm{Hz}$ only in long term recordings). Total power $\left(\mathrm{ms}^{2},<0.4 \mathrm{~Hz}\right)$ is an index of overall variability. The LF/HF ratio and other normalized frequency bands indexes are also

derived (Table 2). By means of the wavelet transform it is possible to estimate the temporal progress of the frequency spectrum of the signal, providing optimal resolution both in time and frequency domain. Indeed, when time domain information is converted into frequency domain information, time-related information is lost, because Fourier transform provides the accumulated power of a given frequency along a period of time, rather than in a given point in time. Wavelet transform has been introduced to HRV analysis to overcome this problem (14).

[Figure 3 here]

The RR time series attractor can be observed by plotting each RR interval against the previous RR interval (Poincaré-plot, figure 4). An attractor is a unique region in the phase-space toward a system tends to evolve, departing for a large set of initial conditions. Once the attractor is constructed, several numerical methods can be applied to estimate its complexity. SD1 reflects short-term HRV while SD2 reflects long-term HRV. Both indexes include linear and nonlinear HRV features (12). The quantification of the statistical entropy features of the system can be performed by measures such as "ApEn" or "SampEn". Basically, they quantify the order of the RR interval time series by taking the logarithm of the probability that patterns being close to each other at the beginning, will remain closer in subsequent observations. Regular sequences will result in lower ApEn values whereas random behavior is associated with larger ApEn values. An increase of entropy values usually reflect parasympathetic predominance situations (12). The detrended fluctuation analysis is a technique that characterizes the pattern of beat to beat variation across multiple scales of measurement, through the short- ( $\alpha 1)$ and long- ( $\alpha 2)$ term fractal correlation scaling exponent alpha. $\alpha 1$ correlates inversely with short- term HRV measurements, while $\alpha 2$ 
correlates inversely with long- term HRV measurements. Values of $\alpha$ close to 0.5 are associated with white noise (no correlation between values), whereas values close to 1.5 are associated with Brownian noise (strong correlation between values). Values near 1 are characteristic of fractal like processes, associated with the dynamic behavior of time series generated by complex systems, such as the autonomic regulation of the sinus rhythm of a healthy subject (Table 3 ) (12).

[Figure 4 here]

HRV indexes are correlated between them. An increase in heart rate, is associated with a reduction in SDNN, RMSSD, HRV power at all frequency bands, entropy values, and attractor dimension, and with an increase in scaling exponent $\alpha$. However, these relations are not linear. The mathematical functions that describe them are different when considering sleep or waking states, which possibly reflects different configurations of ANS functioning (15). In addition, it has been proposed that nonlinear $\mathrm{HRV}$ is a characteristic of the phase transition like dynamics that a healthy human heart rate exhibits between the different behavioral states of the sleep-wake cycle $(16 ; 17)$.

\section{Heart rate variability in selected physiological situations}

\section{Gender related changes of HRV}

HRV measures change during the regular menstrual cycle. SampEn and HF HRV components decrease from the follicular phase to the luteal phase, whereas normalized LF components and LF/HF as well as resting heart rate, increase. SampEn show significant correlations with spectral indexes, free T4 concentrations, and the ratio of estradiol to progesterone concentrations. Thus, the hormonal fluctuations that occur during the luteal phase of the cycle are associated with a shift towards sympathetic prevalence (18). During pregnancy, changes in HRV indexes are observed within the first six weeks after conception, showing a decrease in almost all HRV indexes. It was also found that VLF, LF HF and normalized HF were significantly decreased and LF/HF significantly increased in pregnant subjects in the 3rd trimester 
as compared to the $1 \mathrm{st}$ trimester. The results indicate that during gestation sympathovagal balance shifts progressively from a higher vagal modulation towards a higher sympathetic modulation (19-21). Finally, although HRV is not associated with vasomotor symptoms frequency or intensity in perimenopausal and postmenopausal women, an increase in VLF component is described associated to hot flash periods, which may be involved in the regulatory mechanisms of hot flashes $(22 ; 23)$.

\section{Aging}

Aging is associated with alterations in the neural and endocrine mechanisms that regulate heart rate. Parasympathetic and sympathetic regulation becomes attenuated, renin and angiotensin levels are reduced, and circadian hormonal and temperature rhythms lose amplitude. Consequently, heart rate oscillations show decreased amplitude at all frequency levels with an overall reduction in HRV. Moreover, increasing age is related to alterations in fractal organization of heart beat dynamics as well as to a loss of complex variability. These changes might lead to an impaired ability for stress adaptation and seem to be a common feature with many diseases (24-27).

\section{Cognitive function}

Several studies show that resting autonomic patterns can influence cognitive performance. Possibly, some amount of sympathetic activation reflected by intermediate HRV values would be necessary for optimal cognitive performance. In this regard, it was reported that subjects with higher HF (higher parasympathetic activity) perform better at executive function tasks, probably by concomitant lower anxiety (28). On the other hand, lower resting baroreflex sensitivity, usually associated with a reciprocal reduction of parasympathetic activity and an increase of sympathetic activity, predicted higher values in all parameters of attentional capacity (29). In addition, better performance in a decision-making task was associated with increased LF. The sympathetic prevalence may be necessary to adjust cardiovascular function to cope with increased mental demand (30). Autonomic changes during meditation techniques deserve a separate comment. A prominent peak in absolute LF, not attributed to an increase in sympathetic activity, is usually found in meditation techniques derived from different traditions. Breathing patterns achieved during meditation are associated with a 
synchronization of respiratory and heart rate variations (i.e., cardiorespiratory coupling), which is reflected in this characteristic increase in the LF power (31-33).

\section{Physical activity}

During exercise, the resulting tachycardia is accompanied by a reduction of total HRV power. Thus, although LF absolute values seem unchanged, normalized LF and LF/HR ratio increases reflecting the typical sympathetic activation associated with physical activity (13). This pattern may vary within different subjects, and decrease in nonlinear indexes as SampEn may be more robust for identifying brief physical activity episodes as measured with accelerometers. On the other hand, long-term HRV indexes are relatively stable at various activity levels (34). Of interest, extreme activation of the sympathetic nervous system underlying vigorous exercise lead to a marked reduction in global HRV power and increased nonlinear indexes as SampEn, a pattern also similar to situations with increased propensity to ventricular fibrillation (35). Physical training is associated with higher HRV indexes reflecting vagal predominance which is considered a possible mechanism by which physical activity reduces coronary heart disease risk (36).

\section{Postprandial changes of heart rate variability}

The regional distribution of ANS within the body makes plausible that the same stimulus trigger different responses in different physiological systems, and specifically in cardiac and gastrointestinal activity. In this regard, typical postprandial parasympathetic prevalence within abdominal compartment was associated with a decrease of HF and LF around 30-60 min after a meal, with a concomitant increase in the LF/HF ratio, characteristic of sympathetic predominance (37). The different autonomic configurations of body compartments makes in turn plausible the hypothesis that the disruption of the sleep/wake cycle and other circadian rhythms leads to changes in the balance of the autonomic activity of the thoracic and muscular compartment (towards a predominance of the sympathetic branch) and the intraabdominal compartment (towards a predominance of the parasympathetic branch) (38).

Sleep - wake cycle 
During wakefulness, reflex loops (respiratory sinus arrhythmia, baroreflex and chemoreflexes) and central autonomic network areas (mid cingulate cortex, insula and amygdale) contribute to an increased heart rate, increased sympathetic activity (SNS) and decreased parasympathetic activity (39). In addition, changes in HRV within wake states are associated or predict the operational levels of higher cortical functions, including alertness (40) and decision making (30), suggesting a role of autonomic function in these processes.

Parasympathetic predominance during NREM sleep is characterized by slow EEG rhythms associated with decreased brain activity compared to wakefulness in subcortical (brainstem, thalamus, basal ganglia, basal forebrain) and cortical (prefrontal cortex, anterior cingulate cortex, precuneus) areas, suggesting a lower central command in cardiac autonomic control (39). In this regard, it has been demonstrated that the degree of network connectivity and the strength of physiological interactions between different central and peripheral systems is minimal during slow sleep (41). HRV studies during this sleep stage have revealed that there is a decrease in the LF component and an increase in the HF component relative to wakefulness (42-44). The HF component is strongly associated with changes in the delta EEG band, preceding them in about 12 minutes (45). As for the nonlinear components of HRV, slow wave sleep is characterized as a stage with a higher degree of nonlinear variability in relation to wakefulness, manifested by a lower fractal correlation and a higher degree of entropy (16). These findings are consistent with the parasympathetic prevalence characteristic of this stage and can be interpreted as associated with a decrease in non-reflex central influences (43).

During REM sleep, autonomic cardiac regulation is shared between central control in relation to amygdala activity and homeostatic control of the cardiovascular system by reflex arcs, leading to an increase in heart rate, with sympathetic predominance and decreased parasympathetic activity (39). Consistently, the degree of network connectivity and the strength of physiological interactions between different central and peripheral systems are intermediate between NREM sleep and wakefulness (41). This possibility is supported by the observation that the response of peripheral centers to changes in blood pressure is modified and by the fact that the thermal or electrical stimulation of diencephalon structures is not accompanied by concomitant changes in certain autonomic functions (46). During this stage, a pattern of increase in linear HRV is observed. Studies differ in their reported values for REM sleep, with maxima for the LF component and nulls for the HF component $(42-44 ; 47 ; 48)$. In comparison to wakefulness, no significant changes have been reported in the nonlinear dynamics of 
autonomic cardiac regulation (16;49). Variations in heart rate are particularly marked during the phasic stage of REM sleep, and tend to coincide with ocular movements and with theta activity bursts of this stage (50). Taken together, these findings would reflect the partial release of central modulation on peripheral autonomic activity.

Apart from variations in cardiac autonomic activity associated to sleep stages, an endogenous circadian rhythm has been demonstrated in heart rate and $\mathrm{HRV}$, in the absence of sleep masking effects, general activity, postural changes and light. The results suggest that circadian control of heart rate is not entirely mediated by the sleep - wake cycle and that autonomic modulations are influenced by the circadian regulation. Peak values of this rhythm are seen during late wakefulness for heart rate and during the latter part of the sleep period for HRV measurements. In addition, the administration of melatonin is capable of advancing the endogenous circadian rhythm phase of heart rate and SDNN, RMSSD and HF (51).

\section{Heart rate variability in selected clinical conditions}

\section{Type 2 diabetes mellitus}

The relationship between the presence of diabetes and alterations in cardiac autonomic regulation is well known. This extends to subjects with insulin resistance and to the offspring of type II diabetics patients with no history of diabetes or hypertension where the mean values of HRV are decreased. Low HRV in healthy people is associated with an increase of developing this disorder. In addition, low HRV in patients with diabetes increases the risk of complications and mortality compared with those with normal HRV values. Among other parameters, SampEn and HF HRV are better discriminants to detect autonomic dysfunction. From a circadian point of view, the amplitude of day - night variations in HRV is decreased in subjects with diabetes. These findings support the use of HRV as a risk indicator in type II diabetics (52-54).

\section{Obesity}

Regarding the presence of obesity, a reduction of SDNN, RMSSD and absolute and relative HF HRV is observed, pointing to a relative prevalence of sympathetic activity (55). Circadian alterations of HRV are also described. The LF/HF ratio increases at 
certain points in the afternoon in association with high plasma insulin concentrations (56). Interestingly, increases and decreases in the LF / HF ratio were detected in response to gain and weight reduction achieved through an experimental design (57).

\section{Dyslipidemia}

It has been reported an inverse association between short-term HRV and tryglicerides in elderly people, and between short-term HRV and the LDL/HDL ratio in elderly men but not women (58). A further study demonstrated an inverse relationship between 24 hour HRV indicators reflecting parasympathetic activity (RMSSD) and total cholesterol, LDL and LDL / HDL, adjusted for various demographic and clinical factors including levels of noradrenaline (59).

\section{Hypertension}

ANS disorder, clinically manifested as a hyperkinetic circulation characterized by elevations in heart rate, blood pressure, cardiac output and plasma norepinephrine levels, has been systematically demonstrated in hypertension. Increased sympathetic activity has been reported using spectral analysis of HRV (60). Specifically, compared to control ones, subjects with primary hypertension have decreases in LF and HF components of HRV both during the day as well as the night. The day-night differences in HRV increase after one year of treatment with angiotensin converting enzyme inhibitors (61). In subjects with hypertension and left ventricular hypertrophy (but without coronary pathology), there is a decrease or absence of sleep - wake differences in heart rate, RMSSD, LF and HF, thus evidencing alterations in the circadian rhythm of cardiac autonomic control (62). In addition, flattening of the circadian rhythm of heart rate is independently associated with an increased risk of allcause mortality (63).

\section{Ischemic heart disease}

As described above, existing evidence support the notion that cardiovascular risk factors are associated with altered HRV patterns. Also, autonomic imbalance may provide a unifying framework within which to investigate the impact those factors and other psychosocial determinants on cardiovascular disease (64). When considering 
established cardiovascular disease, impaired HF oscillations of heart rate are the most prominent feature in patients with uncomplicated coronary artery disease. On the other hand, patients with prior myocardial infarction and impaired left ventricular function have a reduced overall heart rate variability, and a specific spectral pattern with a reduced LF spectral component. In addition, patients with prior myocardial infarction have higher ApEn values and lower scaling exponent values, indicating that heart rate dynamics are more random. These measures have revealed to be strong predictors of fatal arrhythmias (65).

\section{Stroke}

Autonomic imbalance has been identified prior to atrial fibrillation (66). Global HRV, quantified by SDNN indicator, is a strong predictor for ischemic stroke development in apparently healthy subjects. The mechanism is unclear, but it is probably due to a decrease in parasympathetic activity that increases the risk of arrhythmias (67). HF HRV is significantly reduced in patients with acute cerebral infarction (68), while elderly survivors of ischemic or hemorrhagic stroke show lower total HRV and LF HRV long after the event (69). In the presence of a hemispheric or trunk stroke, changes similar to those described for coronary disease are observed, where all HRV components are similar during the day and at night, indicating the abolition of circadian rhythmicity of cardiac autonomic regulation (70). HRV differences between hemorrhagic and ischemic stroke are not well established (71).

\section{Mild Cognitive impairment / Alzheimer's disease}

Several works have shown that central components of the ANS, mainly insular cortex and brain stem, are affected in early stages of Alzheimer's disease, manifesting itself as a parasympathetic dysfunction that contributes to the progression of the disease (72). This pattern is maintained in later stages of the disease, where RMSSD and spectral components are lower in patients than in controls. In addition, HRV was found to be significantly correlated with the degree of cognitive impairment (73). 
Autonomic activity may be an important marker in cancer due to its role in modulating inflammation and oxidative stress. HRV indexes have been used in several studies to assess autonomic profiles within this disease. Decreased heart rate variability (mainly reduced SDNN) is associated with shorter survival times in patients with several types of cancer, pointing out that higher vagal nerve activity might play a protective role in cancer (74). Specifically, breast cancer survivors exhibit a decrease in overall variability (SDNN) and parasympathetic activity (RMSSD, HF HRV) when compared to women without the disease (75). Higher SDNN and RMSSD predict lower levels of carcinoembryonic antigen in colorectal cancer patients, supporting the hypothesized role of vagal activity in tumor modulation (76). In prostate cancer patients, SDNN and RMSSD inversely predict PSA levels at 6 and 24 months, being particularly significant in metastatic prostate cancer, indicating stage moderation (77). A study reported that SDNN univariately predicted poor survival in non-small-cell lung cancer (78), while another one found that SDNN and RMSSD predict survival time independently of confounders but only in patients under 65 years old (77).

\section{Anxiety}

Stressors, anxiety, and anxious personality traits are associated with increased cardiac frequency and lower values of parasympathetic HRV indexes during wakefulness, with the effects of stressors and concerns extending to the subsequent nighttime sleep period. These results are independent of other behavioral variables including sleep quality and may mediate the increased cardiovascular risk associated with stress due to the observed reduction in parasympathetic activity (79).

\section{Depression}

Depression has been often reported to be associated with an overall reduction in total HRV, generally characterized by reduced HF HRV and reduced complexity. These are similar findings to those of aging. A reduced HRV characterizes decreased psychological flexibility, emotional dysregulation and defective social engagement, which in turn are linked to hypoactivity of the prefrontal cortex (80). Depression is also recognized as an independent adverse prognostic factor in patients recovering from an acute coronary episode $(81 ; 82)$. Subjects with major depressive disorder have a reduction in $\mathrm{HRV}$ in all frequency components both during the day and during the night. In addition, during the night they present a reduction in nonlinear HRV. Taken together, 
these findings point out a greater sympathetic activation throughout the whole day. It was observed that HRV indicators correlate with sleep quality, but not with depression scores, which shows that sleep disorders typical of depression play an important role in the alteration of autonomic regulation (83).

\section{Schizophrenia}

Schizophrenia is characterized by abnormalities of cortical structures concerned with autonomic control, including prefrontal, cingulated, temporal areas and the hippocampus. Symptom development theories in schizophrenia have long incorporated the notion of autonomic dysfunction, including pupillary, vasomotor, sweating, heart rate, salivation, and temperature changes, most of them suggesting increased sympathetic prevalence as an important feature in the expression of psychosis. HRV pattern in acute schizophrenia is consistent with this notion, since RMSSD index is diminished (84). In stable schizophrenia, subjects exhibit normal autonomic activity at rest and in response to mental stress, but they maintain HRV stress-related changes further than stimulus cessation, in the form of larger relative LF HRV component $(85 ; 86)$.

\section{Social determinants of health}

Extensive research has shown that adverse environmental and working conditions, such as shift work and excessive workload, are related to disease. Psychosocial workload and working time have been associated with low HF HRV (87). In night shift work typical patterns of autonomic predominance are inverted, with increased sympathetic activity during the night (wake period) and increased parasympathetic activity during the day (sleep period), making evident the strong dependence of the autonomic regulation on the sleep-wake cycle (88). When these conditions are maintained for extended periods, sympathetic activity (LF HRV and LF/HF ratio) during sleep in these professionals is greater than that of their colleagues in the morning shift (89). Finally, the lack of exposure to natural light may result in the loss of the circadian rhythm of cardiac autonomic activity, as it was shown in prolonged confinement experiments $(90 ; 91)$. 


\section{Conclusion}

The analysis of the autonomic modulation of heart rate provides information about the state of the ANS in several physiological and clinical situations. It is now widely accepted that the interaction of several biological (genetic, biochemical, biophysical), psychological (personality, mood, behavior), social (family, work, society) and ecological (living environment) factors, plays an important role in the preservation of quality of life and health. The ANS is structural and rhythmically interfaced between forebrain, internal and external environments, to regulate energy, matter and information exchanges. Its overall function is to maintain the body homeostasis and to react predictively or adaptatively to changes in the internal and external environment. All body systems are dependent and affected by the action of others and by external factors in a multilevel and dynamic organization. Thus, the bio-psycho-social nature of the individual is expressed by the function of the ANS, which can be explored by the analysis of the variability of heart rate. In turn, autonomic imbalance, as evidenced by alterations in HRV, may configure a final common pathway to increased morbidity and mortality from a host of conditions and diseases (92-94).

\section{References}

(1) Billman GE. Heart rate variability - a historical perspective. Front Physiol 2011;2:86.

(2) Stauss HM. Heart rate variability. Am J Physiol Regul Integr Comp Physiol 2003 Nov;285(5):R927-R931.

(3) Randall DC, Brown DR, McGuirt AS, Thompson GW, Armour JA, Ardell JL. Interactions within the intrinsic cardiac nervous system contribute to chronotropic regulation. Am J Physiol Regul Integr Comp Physiol 2003 Nov;285(5):R1066-R1075.

(4) Barbieri R, Triedman JK, Saul JP. Heart rate control and mechanical cardiopulmonary coupling to assess central volume: a systems analysis. Am J Physiol Regul Integr Comp Physiol 2002 Nov;283(5):R1210-R1220.

(5) Julien C. The enigma of Mayer waves: Facts and models. Cardiovasc Res $2006 \mathrm{Apr}$ $1 ; 70(1): 12-21$.

(6) Sollers III JJ, Sanford TA, Nabors-Oberg R. Examining changes in HRV in reponse to varying ambient temperature. IEEE Eng Med Biol Mag 2002;21(4):30-4.

(7) Taylor JA, Carr DL, Myers CW, Eckberg DL. Mechanisms underlying very-low-frequency RR-interval oscillations in humans. Circulation 1998 Aug 11;98(6):547-55. 
(8) Grimaldi D, Silvani A, Benarroch EE, Cortelli P. Orexin/hypocretin system and autonomic control: new insights and clinical correlations. Neurology 2014 Jan 21;82(3):271-8.

(9) Mansier P, Clairambault J, Charlotte N, Medigue C, Vermeiren C, LePape G, et al. Linear and non-linear analyses of heart rate variability: a minireview. Cardiovasc Res 1996 Mar;31(3):371-9.

(10) Seely AJ, Macklem PT. Complex systems and the technology of variability analysis. Crit Care 2004 Dec;8(6):R367-R384.

(11) Goldberger AL. Non-linear dynamics for clinicians: chaos theory, fractals, and complexity at the bedside. Lancet 1996 May 11;347(9011):1312-4.

(12) Voss A, Schulz S, Schroeder R, Baumert M, Caminal P. Methods derived from nonlinear dynamics for analysing heart rate variability. Philos Trans A Math Phys Eng Sci 2009 Jan 28;367(1887):277-96.

(13) Task Force of the European Society of Cardiology and the North American Society of Pacing and Electrophysiology. Heart rate variability: standards of measurement, physiological interpretation and clinical use. Circulation 1996 Mar 1;93(5):1043-65.

(14) Pichot V, Gaspoz JM, Molliex S, Antoniadis A, Busso T, Roche F, et al. Wavelet transform to quantify heart rate variability and to assess its instantaneous changes. J Appl Physiol 1999 Mar;86(3):1081-91.

(15) Platisa M, Gal V. Reflection of heart rate regulation on linear and nonlinear heart rate variability measures. Physiol Meas 2006;27:145-54.

(16) Vigo DE, Dominguez J, Guinjoan SM, Scaramal M, Ruffa E, Solerno J, et al. Nonlinear analysis of heart rate variability within independent frequency components during the sleep-wake cycle. Auton Neurosci 2010 Apr 19;154(1-2):84-8.

(17) Kiyono K, Struzik ZR, Aoyagi N, Togo F, Yamamoto Y. Phase transition in a healthy human heart rate. Physical Review Letters 2005;95(058101):1-4.

(18) Bai X, Li J, Zhou L, Li X. Influence of the menstrual cycle on nonlinear properties of heart rate variability in young women. Am J Physiol Heart Circ Physiol 2009 Aug;297(2):H765-H774.

(19) Stein PK, Hagley MT, Cole PL, Domitrovich PP, Kleiger RE, Rottman JN. Changes in 24hour heart rate variability during normal pregnancy. Am J Obstet Gynecol 1999 Apr;180(4):978-85.

(20) Gandhi PH, Mehta HB, Gokhale AV, Desai CB, Gokhale PA, Shah CJ. A study on cardiac autonomic modulation during pregnancy by non-invasive heart rate variability measurement. International Journal of Medicine and Public Health 2014;4(4):441-5.

(21) Matsuo H, Inoue K, Hapsari ED, Kitano K, Shiotani H. Change of autonomic nervous activity during pregnancy and its modulation of labor assessed by spectral heart rate variability analysis. Clin Exp Obstet Gynecol 2007;34(2):73-9. 
(22) Hoikkala H, Haapalahti P, Viitasalo M, Vaananen $H$, Sovijarvi AR, Ylikorkala O, et al. Association between vasomotor hot flashes and heart rate variability in recently postmenopausal women. Menopause 2010 Mar;17(2):315-20.

(23) Jones SM, Guthrie KA, LaCroix AZ, Sternfeld B, Landis CA, Reed SD, et al. Is heart rate variability associated with frequency and intensity of vasomotor symptoms among healthy perimenopausal and postmenopausal women? Clin Auton Res 2016 Feb;26(1):7-13.

(24) lyengar N, Peng CK, Morin R, Goldberger AL, Lipsitz LA. Age-related alterations in the fractal scaling of cardiac interbeat interval dynamics. Am J Physiol 1996 Oct;271(4 Pt 2):R1078-R1084.

(25) Lipsitz LA, Goldberger AL. Loss of 'complexity' and aging. Potential applications of fractals and chaos theory to senescence. JAMA 1992 Apr 1;267(13):1806-9.

(26) Pikkujamsa SM, Makikallio TH, Sourander LB, Raiha IJ, Puukka P, Skytta J, et al. Cardiac interbeat interval dynamics from childhood to senescence : comparison of conventional and new measures based on fractals and chaos theory. Circulation 1999 Jul 27;100(4):393-9.

(27) Vigo DE, Guinjoan SM, Scaramal M, Nicola Siri LN, Cardinali DP. Wavelet transform shows age-related changes of heart rate variability within independent frequency components. Auton Neurosci 2005 Dec 30;123(1-2):94-100.

(28) Hansen AL, Johnsen BH, Thayer JF. Vagal influence on working memory and attention. Int J Psychophysiol 2003 Jun;48(3):263-74.

(29) Duschek S, Muckenthaler M, Werner N, Del Paso GA. Relationships between features of autonomic cardiovascular control and cognitive performance. Biol Psychol 2009 May;81(2):110-7.

(30) Drucaroff L, Kievit R, Guinjoan SM, Gerschcovich ER, Cerquetti D, Leiguarda R, et al. Higher autonomic activation predicts better performance in iowa gambling task. Cogn Behav Neurol 2011 Jun;24(2):93-8.

(31) Peng CK, Henry IC, Mietus JE, Hausdorff JM, Khalsa G, Benson H, et al. Heart rate dynamics during three forms of meditation. Int J Cardiol 2004 May;95(1):19-27.

(32) Cysarz D, Bussing A. Cardiorespiratory synchronization during Zen meditation. Eur J Appl Physiol 2005 Sep;95(1):88-95.

(33) Iglesias SL, Azzara S, Granchetti H, Lagomarsino E, Vigo DE. Anxiety, anger, salivary cortisol and cardiac autonomic activity in palliative care professionals with and without mind-body training experience: Results from a pilot study. European Journal of Integrative Medicine 2014;6(1):98-103.

(34) Hautala AJ, Karjalainen J, Kiviniemi AM, Kinnunen H, Makikallio TH, Huikuri HV, et al. Physical activity and heart rate variability measured simultaneously during waking hours. Am J Physiol Heart Circ Physiol 2010 Mar;298(3):H874-H880. 
(35) Vigo DE, Perez LS, Videla AJ, Perez CD, Hunicken HM, Mercuri J, et al. Heart rate nonlinear dynamics during sudden hypoxia at $8230 \mathrm{~m}$ simulated altitude. Wilderness Environ Med 2010 Mar;21(1):4-10.

(36) Rennie KL, Hemingway $H$, Kumari M, Brunner E, Malik M, Marmot M. Effects of moderate and vigorous physical activity on heart rate variability in a British study of civil servants. Am J Epidemiol 2003 Jul 15;158(2):135-43.

(37) Chang CS, Ko CW, Lien HC, Chou MC. Varying postprandial abdominovagal and cardiovagal activity in normal subjects. Neurogastroenterol Motil 2010 May;22(5):54651, e119.

(38) Cardinali DP, Cano P, Jimenez-Ortega V, Esquifino Al. Melatonin and the metabolic syndrome: physiopathologic and therapeutical implications. Neuroendocrinology 2011;93(3):133-42.

(39) Chouchou F, Desseilles M. Heart rate variability: a tool to explore the sleeping brain? Front Neurosci 2014;8:402.

(40) Chua EC, Tan WQ, Yeo SC, Lau P, Lee I, Mien IH, et al. Heart rate variability can be used to estimate sleepiness-related decrements in psychomotor vigilance during total sleep deprivation. Sleep 2012 Mar;35(3):325-34.

(41) Bashan A, Bartsch RP, Kantelhardt JW, Havlin S, Ivanov PC. Network physiology reveals relations between network topology and physiological function. Nat Commun 2012;3:702.

(42) Otzenberger H, Gronfier C, Simon C, Charloux A, Ehrhart J, Piquard F, et al. Dynamic heart rate variability: a tool for exploring sympathovagal balance continuously during sleep in men. Am J Physiol 1998 Sep;275(3 Pt 2):H946-H950.

(43) Togo F, Yamamoto Y. Decreased fractal component of human heart rate variability during non-REM sleep. Am J Physiol Heart Circ Physiol 2001 Jan;280(1):H17-H21.

(44) Busek P, Vankova J, Opavsky J, Salinger J, Nevsimalova S. Spectral analysis of the heart rate variability in sleep. Physiol Res 2005;54(4):369-76.

(45) Jurysta F, van de Borne P, Migeotte PF, Dumont M, Lanquart JP, Degaute JP, et al. A study of the dynamic interactions between sleep EEG and heart rate variability in healthy young men. Clin Neurophysiol 2003 Nov;114(11):2146-55.

(46) Rector DM, Richard CA, Staba RJ, Harper RM. Sleep states alter ventral medullary surface responses to blood pressure challenges. Am J Physiol Regul Integr Comp Physiol 2000 Apr;278(4):R1090-R1098.

(47) Vanoli E, Adamson PB, Ba L, Pinna GD, Lazzara R, Orr WC. Heart rate variability during specific sleep stages. A comparison of healthy subjects with patients after myocardial infarction. Circulation 1995 Apr 1;91(7):1918-22.

(48) Brandenberger G, Viola AU. Autonomic nervous system activity during sleep in humans. In: Cardinali DP, Pandi-Perumal SR, editors. Neuroendocrine correlates of sleep/wakefulness. 1 ed. New York: Springer; 2006. p. 471-85. 
(49) Viola AU, Tobaldini E, Chellappa SL, Casali KR, Porta A, Montano N. Short-term complexity of cardiac autonomic control during sleep: REM as a potential risk factor for cardiovascular system in aging. PLoS One 2011;6(4):e19002.

(50) Pedemonte $M$, Rodriguez-Alvez A, Velluti RA. Electroencephalographic frequencies associated with heart changes in RR interval variability during paradoxical sleep. Auton Neurosci 2005 Dec 30;123(1-2):82-6.

(51) Vandewalle G, Middleton B, Rajaratnam SM, Stone BM, Thorleifsdottir B, Arendt J, et al. Robust circadian rhythm in heart rate and its variability: influence of exogenous melatonin and photoperiod. J Sleep Res 2007 Jun;16(2):148-55.

(52) Stuckey MI, Petrella RJ. Heart rate variability in type 2 diabetes mellitus. Crit Rev Biomed Eng 2013;41(2):137-47.

(53) Franca da Silva AK, Penachini da Costa de Rezende Barbosa, Marques VF, Destro Christofaro DG, Marques Vanderlei LC. Application of Heart Rate Variability in Diagnosis and Prognosis of Individuals with Diabetes Mellitus: Systematic Review. Ann Noninvasive Electrocardiol 2016 May;21(3):223-35.

(54) Kondo K, Matsubara T, Nakamura J, Hotta N. Characteristic patterns of circadian variation in plasma catecholamine levels, blood pressure and heart rate variability in Type 2 diabetic patients. Diabet Med 2002 May;19(5):359-65.

(55) Rossi RC, Vanderlei LC, Goncalves AC, Vanderlei FM, Bernardo AF, Yamada KM, et al. Impact of obesity on autonomic modulation, heart rate and blood pressure in obese young people. Auton Neurosci 2015 Dec;193:138-41.

(56) Emdin M, Gastaldelli A, Muscelli E, Macerata A, Natali A, Camastra S, et al. Hyperinsulinemia and autonomic nervous system dysfunction in obesity: effects of weight loss. Circulation 2001 Jan 30;103(4):513-9.

(57) Adachi T, Sert-Kuniyoshi FH, Calvin AD, Singh P, Romero-Corral A, van der Walt C, et al. Effect of weight gain on cardiac autonomic control during wakefulness and sleep. Hypertension 2011 Apr;57(4):723-30.

(58) Greiser KH, Kluttig A, Schumann B, Swenne CA, Kors JA, Kuss O, et al. Cardiovascular diseases, risk factors and short-term heart rate variability in an elderly general population: the CARLA study 2002-2006. Eur J Epidemiol 2009;24(3):123-42.

(59) Thayer JF, Fischer JE. Heart rate variability, overnight urinary norepinephrine, and plasma cholesterol in apparently healthy human adults. Int J Cardiol 2013 Jan 20;162(3):240-4.

(60) Palatini P, Julius S. The role of cardiac autonomic function in hypertension and cardiovascular disease. Curr Hypertens Rep 2009 Jun;11(3):199-205.

(61) Kolasinska-Kloch W, Furgala A, Banach T, Laskiewicz J, Thor PJ. Circadian heart rate variability in patients with primary arterial hypertension. Przegl Lek 2002;59(9):752-5.

(62) Chakko S, Mulingtapang RF, Huikuri HV, Kessler KM, Materson BJ, Myerburg RJ. Alterations in heart rate variability and its circadian rhythm in hypertensive patients 
with left ventricular hypertrophy free of coronary artery disease. Am Heart J 1993 Dec;126(6):1364-72.

(63) Verdecchia P, Schillaci G, Borgioni C, Ciucci A, Telera MP, Pede S, et al. Adverse prognostic value of a blunted circadian rhythm of heart rate in essential hypertension. J Hypertens 1998 Sep;16(9):1335-43.

(64) Thayer JF, Yamamoto SS, Brosschot JF. The relationship of autonomic imbalance, heart rate variability and cardiovascular disease risk factors. Int J Cardiol 2010 May 28;141(2):122-31.

(65) Huikuri HV, Makikallio TH. Heart rate variability in ischemic heart disease. Auton Neurosci 2001 Jul 20;90(1-2):95-101.

(66) Gallo C, Bocchino PP, Magnano M, Gaido L, Zema D, Battaglia A, et al. Autonomic Tone Activity Before the Onset of Atrial Fibrillation. J Cardiovasc Electrophysiol 2016 Dec 14.

(67) Binici Z, Mouridsen MR, Kober L, Sajadieh A. Decreased nighttime heart rate variability is associated with increased stroke risk. Stroke 2011 Nov;42(11):3196-201.

(68) Kuriyama N, Mizuno T, Niwa F, Watanabe $\mathrm{Y}$, Nakagawa M. Autonomic nervous dysfunction during acute cerebral infarction. Neurol Res 2010 Oct;32(8):821-7.

(69) McLaren A, Kerr S, Allan L, Steen IN, Ballard C, Allen J, et al. Autonomic function is impaired in elderly stroke survivors. Stroke 2005 May;36(5):1026-30.

(70) Korpelainen JT, Sotaniemi KA, Huikuri HV, Myllyla VV. Circadian rhythm of heart rate variability is reversibly abolished in ischemic stroke. Stroke 1997 Nov;28(11):2150-4.

(71) Yperzeele L, van Hooff RJ, Nagels G, De SA, De KJ, Brouns R. Heart rate variability and baroreceptor sensitivity in acute stroke: a systematic review. Int J Stroke 2015 Aug;10(6):796-800.

(72) Collins O, Dillon S, Finucane C, Lawlor B, Kenny RA. Parasympathetic autonomic dysfunction is common in mild cognitive impairment. Neurobiol Aging 2012 Oct;33(10):2324-33.

(73) Zulli R, Nicosia F, Borroni B, Agosti C, Prometti P, Donati P, et al. QT dispersion and heart rate variability abnormalities in Alzheimer's disease and in mild cognitive impairment. J Am Geriatr Soc 2005 Dec;53(12):2135-9.

(74) Zhou X, Ma Z, Zhang L, Zhou S, Wang J, Wang B, et al. Heart rate variability in the prediction of survival in patients with cancer: A systematic review and meta-analysis. J Psychosom Res 2016 Oct;89:20-5.

(75) Palma MR, Vanderlei LC, Ribeiro FE, Mantovani AM, Christofaro DG, Fregonesi CE. The relationship between post-operative time and cardiac autonomic modulation in breast cancer survivors. Int J Cardiol 2016 Dec 1;224:360-5.

(76) Mouton C, Ronson A, Razavi D, Delhaye F, Kupper N, Paesmans M, et al. The relationship between heart rate variability and time-course of carcinoembryonic antigen in colorectal cancer. Auton Neurosci 2012 Jan 26;166(1-2):96-9. 
(77) De CM, van BD, Schallier D, De GJ, Gidron Y. The relationship between vagal nerve activity and clinical outcomes in prostate and non-small cell lung cancer patients. Oncol Rep 2013 Nov;30(5):2435-41.

(78) Kim K, Chae J, Lee S. The Role of Heart Rate Variability in Advanced Non-Small-Cell Lung Cancer Patients. J Palliat Care 2015;31(2):103-8.

(79) Brosschot JF, Van DE, Thayer JF. Daily worry is related to low heart rate variability during waking and the subsequent nocturnal sleep period. Int J Psychophysiol 2007 Jan;63(1):39-47.

(80) Sgoifo A, Carnevali L, Alfonso ML, Amore M. Autonomic dysfunction and heart rate variability in depression. Stress 2015;18(3):343-52.

(81) Carney RM, Saunders RD, Freedland KE, Stein P, Rich MW, Jaffe AS. Association of depression with reduced heart rate variability in coronary artery disease. Am J Cardiol 1995 Sep 15;76(8):562-4.

(82) Vigo DE, Nicola Siri L, Ladron De Guevara MS, Martinez-Martinez JA, Fahrer RD, Cardinali DP, et al. Relation of depression to heart rate nonlinear dynamics in patients $>$ or $=60$ years of age with recent unstable angina pectoris or acute myocardial infarction. Am J Cardiol 2004 Mar 15;93(6):756-60.

(83) Yang AC, Tsai SJ, Yang CH, Kuo CH, Chen TJ, Hong CJ. Reduced physiologic complexity is associated with poor sleep in patients with major depression and primary insomnia. J Affect Disord 2011 Jun;131(1-3):179-85.

(84) Boettger S, Hoyer D, Falkenhahn K, Kaatz M, Yeragani VK, Bar KJ. Altered diurnal autonomic variation and reduced vagal information flow in acute schizophrenia. Clin Neurophysiol 2006 Dec;117(12):2715-22.

(85) Castro MN, Vigo DE, Weidema H, Fahrer RD, Chu EM, de Achaval D, et al. Heart rate variability response to mental arithmetic stress in patients with schizophrenia Autonomic response to stress in schizophrenia. Schizophr Res 2007 Oct 1.

(86) Castro MN, Vigo DE, Chu EM, Fahrer RD, de AD, Costanzo EY, et al. Heart rate variability response to mental arithmetic stress is abnormal in first-degree relatives of individuals with schizophrenia. Schizophr Res 2009 Apr;109(1-3):134-40.

(87) Togo F, Takahashi M. Heart rate variability in occupational health --a systematic review. Ind Health 2009 Dec;47(6):589-602.

(88) Ito H, Nozaki M, Maruyama T, Kaji $Y$, Tsuda $Y$. Shift work modifies the circadian patterns of heart rate variability in nurses. Int J Cardiol 2001 Jul;79(2-3):231-6.

(89) Chung MH, Kuo TB, Hsu N, Chu H, Chou KR, Yang CC. Sleep and autonomic nervous system changes - enhanced cardiac sympathetic modulations during sleep in permanent night shift nurses. Scand J Work Environ Health 2009 May;35(3):180-7.

(90) Vigo DE, Ogrinz B, Wan L, Bersenev E, Tuerlinckx F, Van den Bergh O, et al. Sleep-wake differences in heart rate variability during a 105-day simulated mission to Mars. Aviat Space Environ Med 2012 Feb;83(2):125-30. 
(91) Vigo DE, Tuerlinckx F, Ogrinz B, Wan L, Simonelli G, Bersenev E, et al. Circadian rhythm of autonomic cardiovascular control during Mars500 simulated mission to Mars. Aviat Space Environ Med 2013 Oct;84(10):1023-8.

(92) Recordati G. A thermodynamic model of the sympathetic and parasympathetic nervous systems. Auton Neurosci 2003 Jan 31;103(1-2):1-12.

(93) Moser M, Fruhwirth M, Penter R, Winker R. Why life oscillates--from a topographical towards a functional chronobiology. Cancer Causes Control 2006 May;17(4):591-9.

(94) Cardinali D. Autonomic nervous system. Basic and clinical aspects. Cham: Springer International Publishing (in press); 2017. 
Table 1: Selected time domain HRV indexes

\begin{tabular}{lll}
\hline Index & Definition & Interpretation \\
\hline RRM (ms) & Mean duration of RR intervals. & Reciprocal of mean heart rate. \\
SDNN (ms) & $\begin{array}{l}\text { Standard deviation of all RR } \\
\text { intervals. }\end{array}$ & Coarse quantification of HRV. \\
RMSSD (ms) & $\begin{array}{l}\text { Square root of the mean of the } \\
\text { squared differences between } \\
\text { adjacent RR intervals. }\end{array}$ & $\begin{array}{l}\text { High frequency variations of HRV } \\
\text { of parasympathetic origin. }\end{array}$ \\
& & \\
\hline
\end{tabular}


Table 2: Selected frequency domain HRV indexes

\begin{tabular}{|c|c|c|}
\hline Index & Definition & Interpretation \\
\hline $\mathrm{TP}\left(\mathrm{ms}^{2}\right)$ & Total power $(<0.4 \mathrm{~Hz})$ & Coarse quantification of HRV \\
\hline ULF (ms2) & $\begin{array}{l}\text { Power in the ultra low frequency range }(< \\
0.0033 \mathrm{~Hz}) \text {. Only in long term recordings. }\end{array}$ & Circadian HRV variations. \\
\hline $\operatorname{VLF}\left(\mathrm{ms}^{2}\right)$ & $\begin{array}{l}\text { Power in the very low frequency range }(< \\
0.04 \mathrm{~Hz} \text { in short term recordings or } 0.0033 \\
\mathrm{~Hz}-0.04 \mathrm{~Hz} \text { in long term recordings) }\end{array}$ & $\begin{array}{l}\text { Possibly originated in hormonal } \\
\text { factors and peripheral } \\
\text { thermoregulation. Depends mainly } \\
\text { on parasympathetic outflow. }\end{array}$ \\
\hline $\mathrm{LF}\left(\mathrm{ms}^{2}\right)$ & $\begin{array}{l}\text { Power in the low frequency range }(0.04 \mathrm{~Hz}- \\
0.15 \mathrm{~Hz}) \text {. }\end{array}$ & $\begin{array}{l}\text { Baroreflex. Depends on sympathetic } \\
\text { and parasympathetic outflow. }\end{array}$ \\
\hline $\mathrm{HF}\left(\mathrm{ms}^{2}\right)$ & $\begin{array}{l}\text { Power in the high frequency range }(0.15 \mathrm{~Hz}- \\
0.4 \mathrm{~Hz}) \text {. }\end{array}$ & $\begin{array}{l}\text { Cardiopulmonary reflex. Depends } \\
\text { on parasympathetic outflow. }\end{array}$ \\
\hline ULF (\%) & $\begin{array}{l}\text { ULF power in percentage units ULF }(\%)= \\
100 \times \text { ULF/ total power. Only in long term } \\
\text { recordings. }\end{array}$ & Relative contribution of ULF HRV. \\
\hline $\operatorname{VLF}(\%)$ & $\begin{array}{l}\text { VLF power in percentage units VLF }(\%)= \\
100 \times \text { VLF/ TP }\end{array}$ & Relative contribution of VLF HRV. \\
\hline LF (\%) & $\begin{array}{l}\text { LF power in percentage units LF }(\%)=100 X \\
\text { LF/ TP }\end{array}$ & Relative contribution of LF HRV. \\
\hline $\mathrm{HF}(\%)$ & $\begin{array}{l}\text { HF power in percentage units HF }(\%)=100 \\
X \mathrm{HF} / \mathrm{TP}\end{array}$ & Relative contribution of HF HRV. \\
\hline LF (n.u.) & $\begin{array}{l}\text { LF power in normalized units LF (n.u.) }=100 \\
X \mathrm{LF} /(\mathrm{LF}+\mathrm{HF})\end{array}$ & $\begin{array}{l}\text { Relative contribution of LF HRV } \\
\text { within LF + HF range. }\end{array}$ \\
\hline HF (n.u.) & $\begin{array}{l}\text { HF power in normalized units HF (n.u.) }=100 \\
X \mathrm{HF} /(\mathrm{LF}+\mathrm{HF})\end{array}$ & $\begin{array}{l}\text { Relative contribution of HF HRV } \\
\text { within } \mathrm{LF}+\mathrm{HF} \text { range. }\end{array}$ \\
\hline LF/HF & Low frequency / high frequency ratio & $\begin{array}{l}\text { Considered to reflect sympathetic - } \\
\text { parasympathetic balance }\end{array}$ \\
\hline
\end{tabular}


Table 3: Selected nonlinear HRV indexes

\begin{tabular}{|c|c|c|}
\hline Index & Definition & Interpretation \\
\hline SD1, SD2 & $\begin{array}{l}\text { Dispersion of the points } \\
\text { around the minor (SD1) } \\
\text { and major (SD2) axis of } \\
\text { an ellipse fitted to the } \\
\text { attractor. }\end{array}$ & $\begin{array}{l}\text { Set of numerical values toward which a system } \\
\text { tends to evolve. SD1 reflects short-term HRV } \\
\text { while SD2 reflects long-term HRV. Both indexes } \\
\text { include linear and nonlinear HRV features. }\end{array}$ \\
\hline Apen, Sampen & $\begin{array}{l}\text { Entropy measurement of } \\
\text { heart - beat time series. }\end{array}$ & $\begin{array}{l}\text { Random behavior is associated with larger ApEn } \\
\text { values. Usually reflects parasympathetic } \\
\text { predominance. }\end{array}$ \\
\hline $\begin{array}{l}\text { Short }(\alpha 1) \text { and } \\
\text { long }(\alpha 2) \text { term } \\
\text { scaling exponent } \\
\text { alpha }\end{array}$ & $\begin{array}{l}\text { Fractal correlation } \\
\text { properties of heart - beat } \\
\text { time series as calculated } \\
\text { by detrended fluctuation } \\
\text { analysis. }\end{array}$ & $\begin{array}{l}\text { Highly correlated heart beat time series will } \\
\text { result in higher scaling exponent alpha. } \alpha 1 \\
\text { correlates inversely with short- term HRV } \\
\text { measurements, while } \alpha 2 \text { correlates inversely } \\
\text { with long- term HRV measurements. Values of } \alpha \\
\text { close to } 0.5 \text { are associated with white noise, } \\
\text { whereas values close to } 1.5 \text { are associated with } \\
\text { Brownian noise. Values near } 1 \text { are characteristic } \\
\text { of the dynamic behavior of complex systems. }\end{array}$ \\
\hline
\end{tabular}




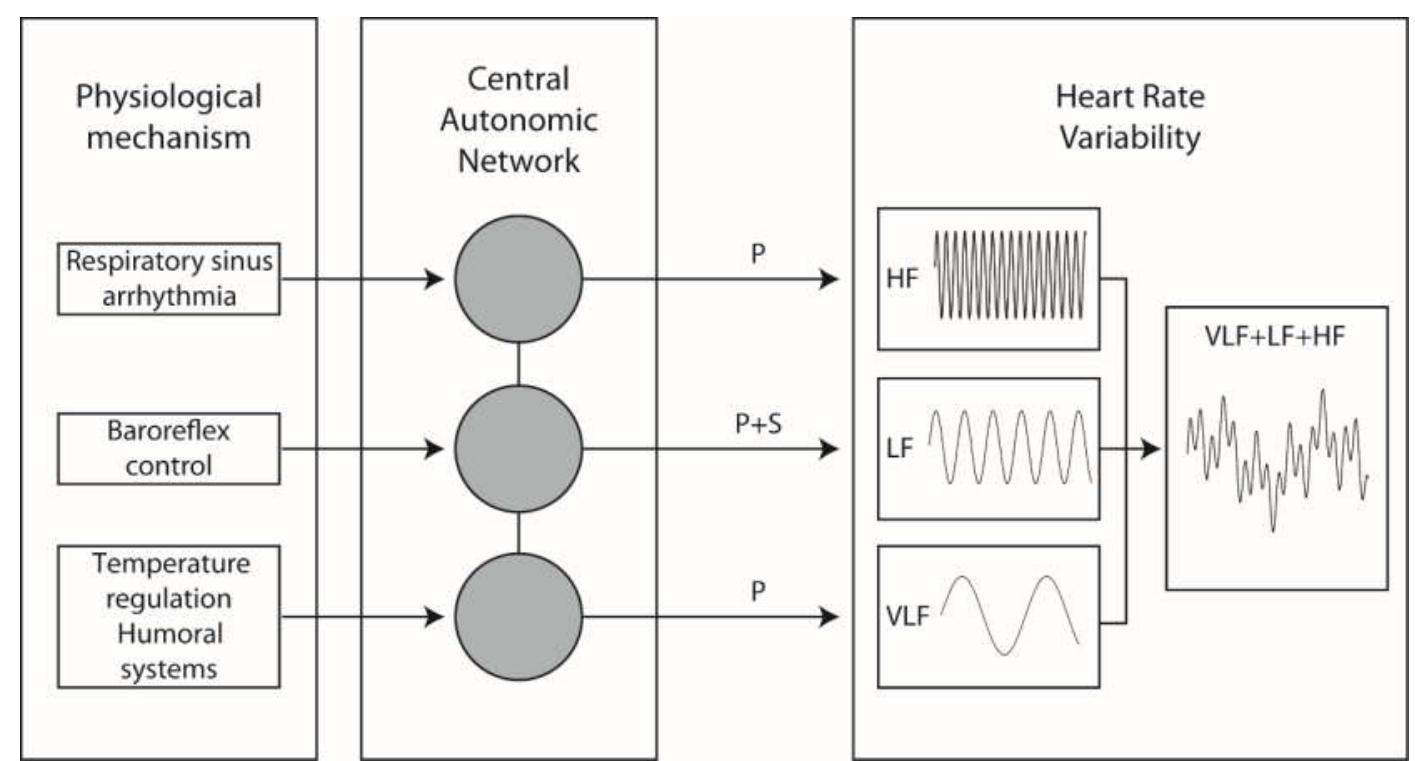

Figure 1: Physiological mechanisms underlying heart rate variability. Respiratory rhythm is associated with high frequency changes of heart rate, mediated by parasympathetic activity; the baroreflex function is associated with low frequency changes of heart rate, mediated by sympathetic and parasympathetic activity; less settled processes like temperature regulation or endocrine systems are related with very low frequency changes of heart rate, with dependence on the presence of parasympathetic outflow. P: parasympathetic; S: sympathetic; VLF: very low frequency; LF: low frequency; HF high frequency. Reproduced with permission from (90).

[The figure was designed by Daniel Vigo, but permission for publication should be requested to the journal where originally appeared] 


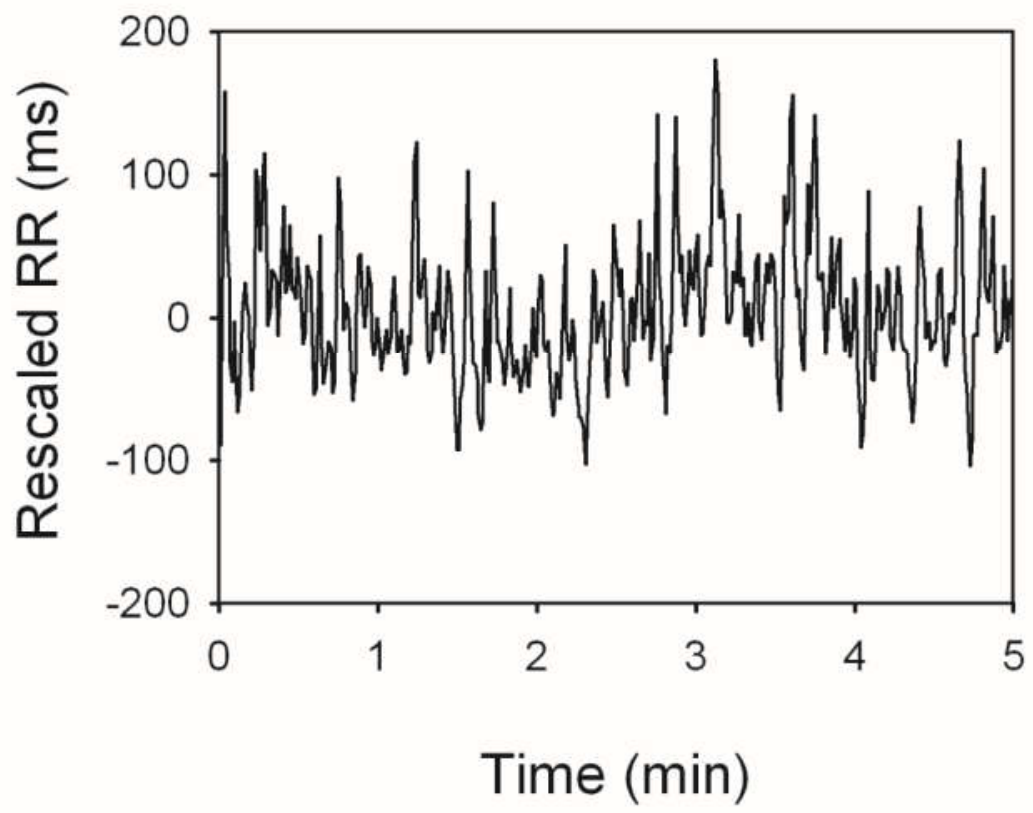

Figure 2: Time domain HRV. Five minutes section of a HRV recording of a young healthy subject. The mean RR interval was subtracted from the original data. 


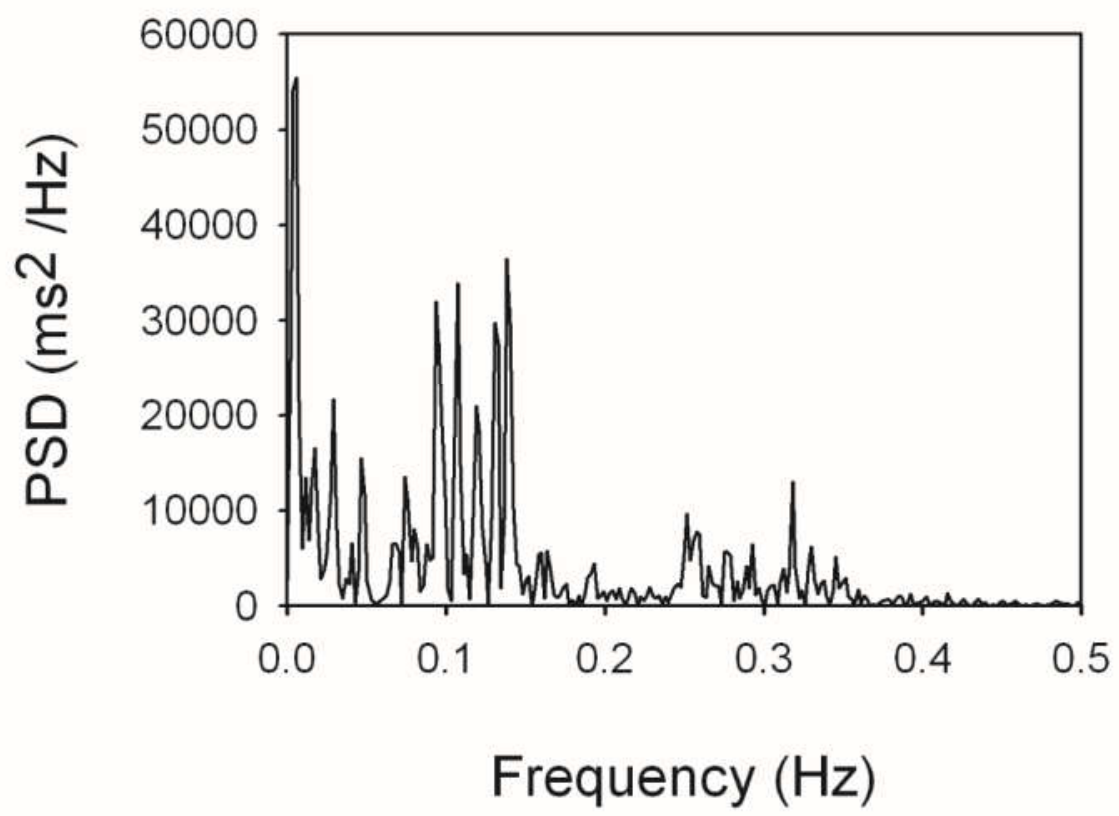

Figure 3: Frequency domain HRV. Spectral density of the same individual of figure 2. Peaks are observed around $0.3 \mathrm{~Hz}$ (high frequency), $0.1 \mathrm{~Hz}$ (low frequency), and < $0.04 \mathrm{~Hz}$ (very low frequency). 


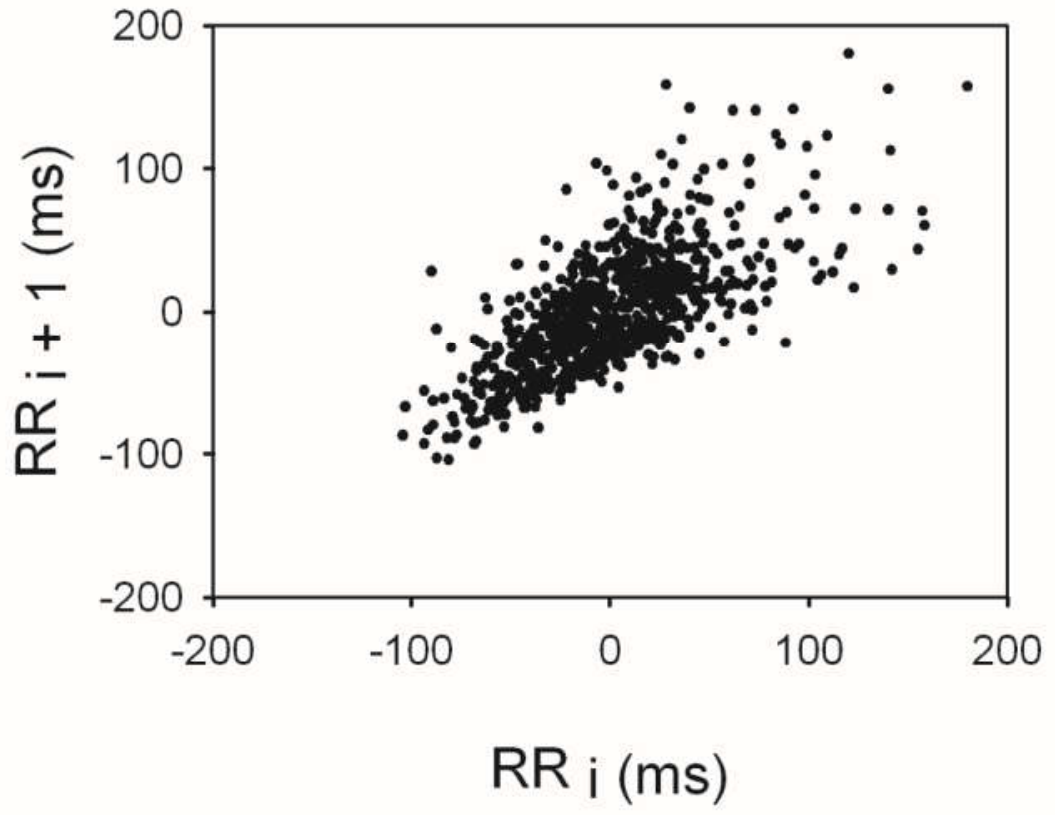

Figure 4: Poincaré Plot of the same individual of figure 2. RR intervals are depicted as a function of the previous RR intervals, composing the heart rate attractor. 